\title{
Inversion of velocity parameters in multilayered elliptical anisotropy medium - synthetic data example
}

\author{
Bartosz Gierlach*, and Tomasz Danek \\ AGH University of Science and Technology, Department of Geoinformatics and Applied Computer \\ Sciences, Cracow, Poland
}

\begin{abstract}
In the medium composed of parallel sedimentary layers, model of elliptic anisotropy is often better approximation of real conditions than simple layers with constant velocity. In such model a velocity is described with 3 parameters: $a, b$ (responsible for vertical velocity and its gradient) and $\chi$ - elliptic anisotropy coefficient, describing changes of velocity with direction of wave propagation. In this case, seismic rays are no longer straight lines but elliptic-shaped curves. In this contribution we present a tool for inversion of velocity parameters in multilayered medium considering model of elliptic anisotropy. Input data contains measured traveltimes of direct wave between all sources and receivers. The depth of boundaries and existence of elliptic anisotropy in specific layers are not subject of inversion. In order to obtain each synthetic traveltime, necessary for the target function, ray tracing must be carried out using Fermat's principle directly. The optimization in both parameters inversion and ray tracing is local and performed with Simplex algorithm from GSL library. The tests of the tool was conducted on the synthetic data with various types of start model containing one or more layers with or without elliptic anisotropy.
\end{abstract}

Keywords: seismic method, ray tracing, inversion, elastic anisotropy

\section{Introduction}

The basic model used in interpretation of seismic data is a set of parallel layers having constant velocity. In the real geological conditions there is a common occurrence of horizontal sedimentary layers in which velocity varies inside a layer. These changes can be approximated with a velocity gradient (linear change with depth) and an elliptical anisotropy (change with direction of wave propagation).

Description of the medium in such way demands additional parameters. It can be treated as an advantage in context of model accuracy, but on the other hand additional parameters may make the inversion process more difficult. The inversion of velocity parameters with

\footnotetext{
* Corresponding author: gierlach@agh.edu.pl
} 
assumption of vertical gradient and elliptical anisotropy requires also optimization of ray path as their trajectory is arc-shaped.

The decision about optimal number of parameters should be made in the way that ensures balance between the fit of modelled traveltimes with measurements and the simplicity of the model. In this paper we used the Bayesian Information Criterion for choosing parameterization for specific dataset with respect to resolving power of the data.

\section{Mathematic background}

\subsection{Model of elliptical anisotropy}

In this paper, we consider model of Hookean solid described with 3 parameters per layer. A linear increase of velocity with depth is described with $a$ and $b$ parameters (velocity at zero depth and vertical velocity gradient, respectively), while elliptical anisotropy parameter $\chi$ connects vertical $\left(v_{z}\right)$ and horizontal $\left(v_{x}\right)$ velocities [1]:

$$
\chi=\frac{v_{x}^{2}+v_{z}^{2}}{2 v_{z}^{2}}
$$

The elliptical anisotropy model is simplification of model with Thomsen's constants $(\delta, \varepsilon)$ for the condition $\delta=\varepsilon$ as defined in [2] (see also [3]). It is characterized by elliptical wavefronts propagating from the source. The elliptical nature of the wavefront surface and the group velocity $v_{S H}$ as a function of group angle $\Phi$ is shown in the equation (4):

$$
\frac{1}{v_{S H}^{2}(\Phi)}=\frac{\cos ^{2} \Phi}{\beta^{2}}+\frac{\sin ^{2} \Phi}{(1+2 \gamma) \beta^{2}}
$$

For the near-vertical incidence of seismic ray (short-spread approximation) one can use mentioned equations also to obtain velocity of P-waves propagating along the angle $\theta$ [4]:

$$
v(\theta)=(a+b z) \sqrt{1+2 \chi \sin ^{2} \theta}
$$

Thus, we obtain model with ab $\chi$ parametrization used for traveltime calculation. With the source at $(0,0)$ and receiver at $(x, z)$ the traveltime of a signal propagating along the elliptic ray is given with formula[5]:

$$
t=\frac{1}{b}\left\{\operatorname{atanh}\left[p b x-\sqrt{1-(1+2 \chi) p^{2} a^{2}}\right]+\operatorname{atanh} \sqrt{1-(1+2 \chi) p^{2} a^{2}}\right\}
$$

where

$$
p=\frac{2 x}{\sqrt{\left[x^{2}+(1+2 \chi) z^{2} \llbracket(2 a+b z)^{2}(1+2 \chi)+b^{2} x^{2}\right]}}
$$

The mentioned equations are proper for model composed of one layer. The analytical solution for traveltime in 2-layers model exists and is described in [5]. For the multilayered model, when one needs to find several points where ray crosses the boundaries, there is a need to perform optimization of ray path. Since the rays are not straight lines one cannot use solutions 
based on conventional Snell's law. In our method we use directly Fermat's principle (principle of least time) that the path taken between two points by a ray is the path that can be traversed in the least time.

\subsection{Simplex optimization}

A method proposed by Nelder and Mead [6] can be used for minimization of function of $n$ variables. It does not use gradients thus can be used also for not differentiable functions.

Simplex in $n$-dimensional space of solutions is set of $n+1$ points creating polyhedron. During one iteration of the optimization function values at $n+1$ vertices of a simplex are calculated and vertex with the worst value is replaced by another point. The operations used to find new vertex of the polyhedron are reflection, expansion and contraction with respect to the symmetry centre of polyhedron, excluding discarded point. For more details, see [7].

\subsection{Bayesian Information Criterion}

In geophysical investigations statistical approach is often important for right interpretation of data. Traveltimes are always burdened with measurement errors and small-scale differences in elastic properties. Some of the velocity parameters can lose their importance for the result due to these errors which means that simpler model would be better to describe the medium.

One of the measures which allows one to choose optimal model is Bayesian Information Criterion (BIC) [8]. Under the assumption of normal distribution of the model errors or disturbances. The BIC value can be calculated as [9]:

$$
B I C=n \ln \left(\sigma_{e}^{2}\right)+k \ln (n)
$$

where $k$ is the number of parameters in the model,$n$ is the number of data points and $\sigma_{\mathrm{e}}^{2}$ is the sum of squared residuals. It should be minimized to ensure that assumed model gives the best balance between simplicity and fit to data. BIC value is larger either if the differences between measured and modelled values are larger or there is more parameters necessary to describe the model. Penalization for additional parameters is relatively large so BIC is more critical for the model over-parameterization if compared with another information criterions (i.e. Akaike) and is widely used in similar researches (see [10]).

\section{Numerical example}

Let us consider two scenarios - Model 1 is composed of three layers with the middle one anisotropic and Model 2 has five layers with forth layer anisotropic (see Table.1). The aim of the research was to test software for velocity parameters inversion and find the highest level of error for which adding elliptical anisotropy coefficient to the velocity model is justified.

\subsection{Software description}

In the research process we used our software written in C language and operating on Linux platform. The program has 3 working modes: forward modelling, single source and multiple source inversion. During the optimization process the number of layers and layers with allowed anisotropy and gradient are invariable. 
Table 1. Velocity models used for the research.

\begin{tabular}{|c|c|c|c|}
\hline \multicolumn{2}{|c|}{ Model 1 } & \multicolumn{2}{c|}{ Model2 } \\
\hline Velocity & Bottom depth & Velocity & Bottom depth \\
\hline $\mathrm{v}=2000 \mathrm{~m} / \mathrm{s}$ & $1500 \mathrm{~m}$ & $\mathrm{v}=2000 \mathrm{~m} / \mathrm{s}$ & $1200 \mathrm{~m}$ \\
\hline $\mathrm{a}=2500 \mathrm{~m} / \mathrm{s} \mathrm{b}=0.2 \mathrm{~s}^{-1} \chi=0.06$ & $2600 \mathrm{~m}$ & $\mathrm{v}=2500 \mathrm{~m} / \mathrm{s}$ & $1900 \mathrm{~m}$ \\
\hline $\mathrm{v}=4500 \mathrm{~m} / \mathrm{s}$ & & $\mathrm{v}=3200 \mathrm{~m} / \mathrm{s}$ & $2200 \mathrm{~m}$ \\
\hline \multirow{2}{*}{} & & $\mathrm{a}=3000 \mathrm{~m} / \mathrm{s} \mathrm{b}=0.2 \mathrm{~s}^{-1} \chi=0.04$ & $2600 \mathrm{~m}$ \\
\cline { 3 - 4 } & $\mathrm{v}=4500 \mathrm{~m} / \mathrm{s}$ & \multicolumn{1}{|c}{} \\
\cline { 3 - 4 } & &
\end{tabular}

Optimization of velocity model can be performed separately for each source or for several sources together. The target function is the sum of squared differences between modelled and measured traveltimes. For each velocity model and each pair of source and receiver the optimization of ray path is required. It is performed according to the Fermat's principle thus the target function is the traveltime for specific velocity model.

Both optimization of velocity model and ray path are carried out with Nelder-Mean simplex algorithm. The start steps of optimization are chosen arbitrarily and the stop criterion is based on distance between vertices.

\subsection{Research outline}

The first step of the procedure was generation of synthetic datasets basing on velocity models mentioned above and measurement sets composed of 40 geophones located inside the borehole at the depth in range between $2400 \mathrm{~m}$ and $2900 \mathrm{~m}$ below the surface. The waves were simulated by 10 sources at the surface located along the line with $200 \mathrm{~m}$ spacing (between $200 \mathrm{~m}$ and $2000 \mathrm{~km}$ from the borehole). To simulate real conditions of measurements a random noise (normally distributed) was added to modelled traveltimes. The amplitudes of the noise was different, with standard deviations in range from $0.1 \%$ to $2 \%$ of mean traveltime.

For each dataset multiple source inversion was carried out with different number of model parameters. The simplest model has only constant velocities ( 3 or 5 parameters) while more complicated models has up to 9 or 11 parameters (isotropic layers treated as they would be anisotropic). Then BIC value was calculated for each variant of the start model to learn what is the optimal number of parameters for a given level of random noise.

\subsection{Results}

For each modelled set of traveltimes, inversion of velocity parameters was carried out successfully. Points where ray paths cross subsequent boundaries were calculated for each pair of source and receiver and can be displayed with our program (Fig. 1). Note that in the anisotropic layer path is displayed for simplicity as a straight line while actually it is arcshaped according to the equation (4). Explicit formula for the ray path is presented in [10]. 


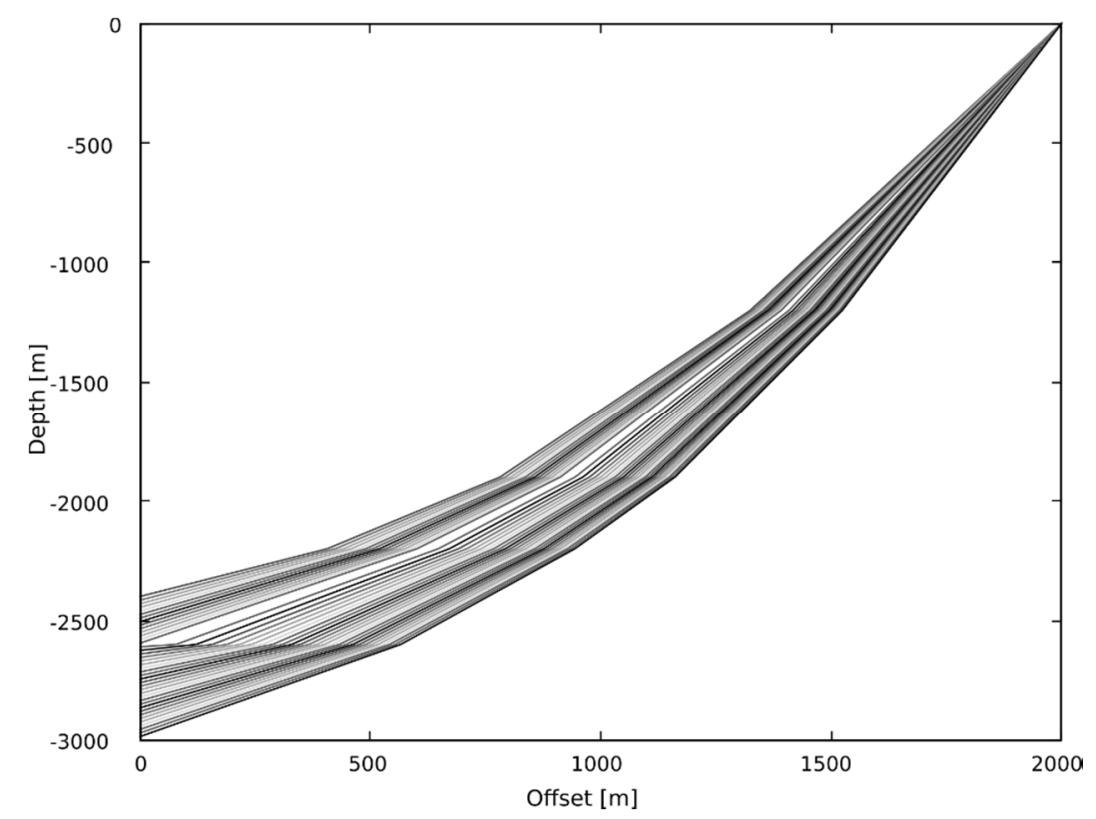

Fig. 1. Ray paths for source \#10 and all receivers for 5-layers model.

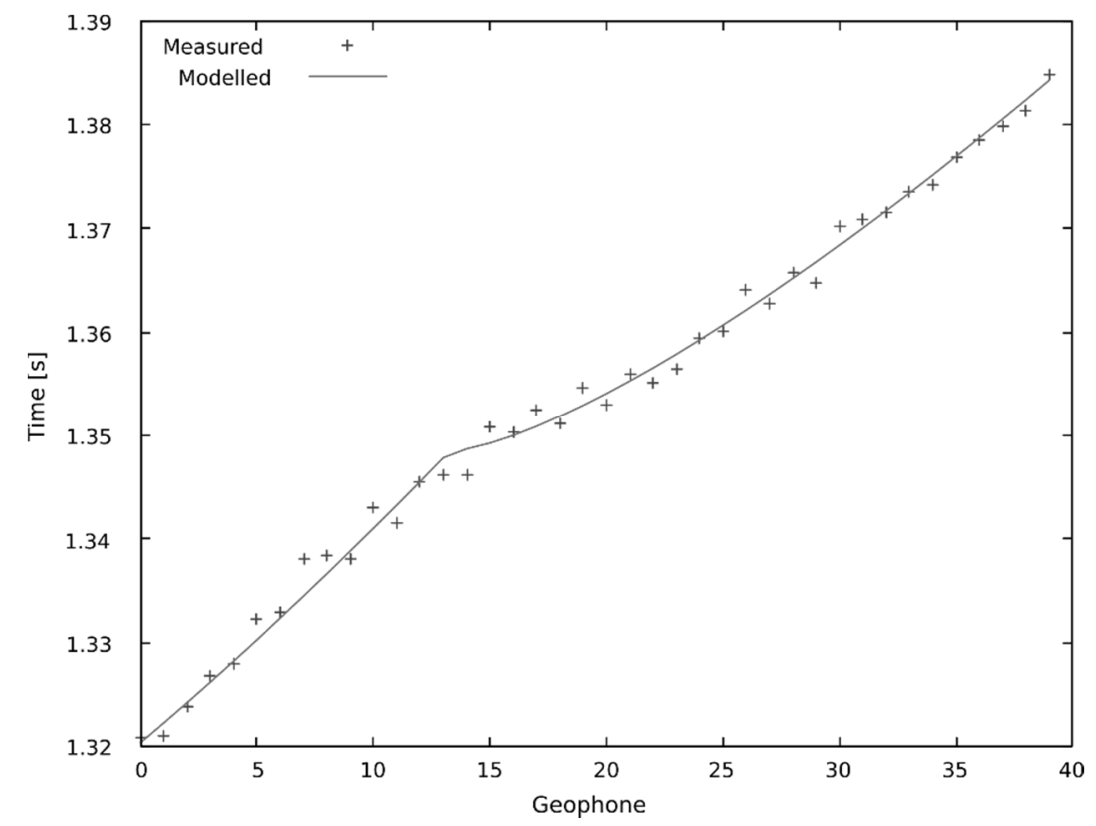

Fig. 2. Comparison of the modelled and disturbed model ("measured") traveltimes for source \#10 with $0.1 \%$ noise (5 layers model).

The traveltimes obtained with inversion are in agreement with measured time for all variants (Fig. 2). For the number of parameters equal to that used for modelling (4 or 6 parameters) obtained velocity model is quite consistent with the simulated values. If there is less parameters, changes of other values compensate lack of anisotropy or gradient (though target function has greater value). If the number of parameters is greater than in simulation, the velocity model may contain some artificial anisotropy or gradient but it results from the added random deviations. 

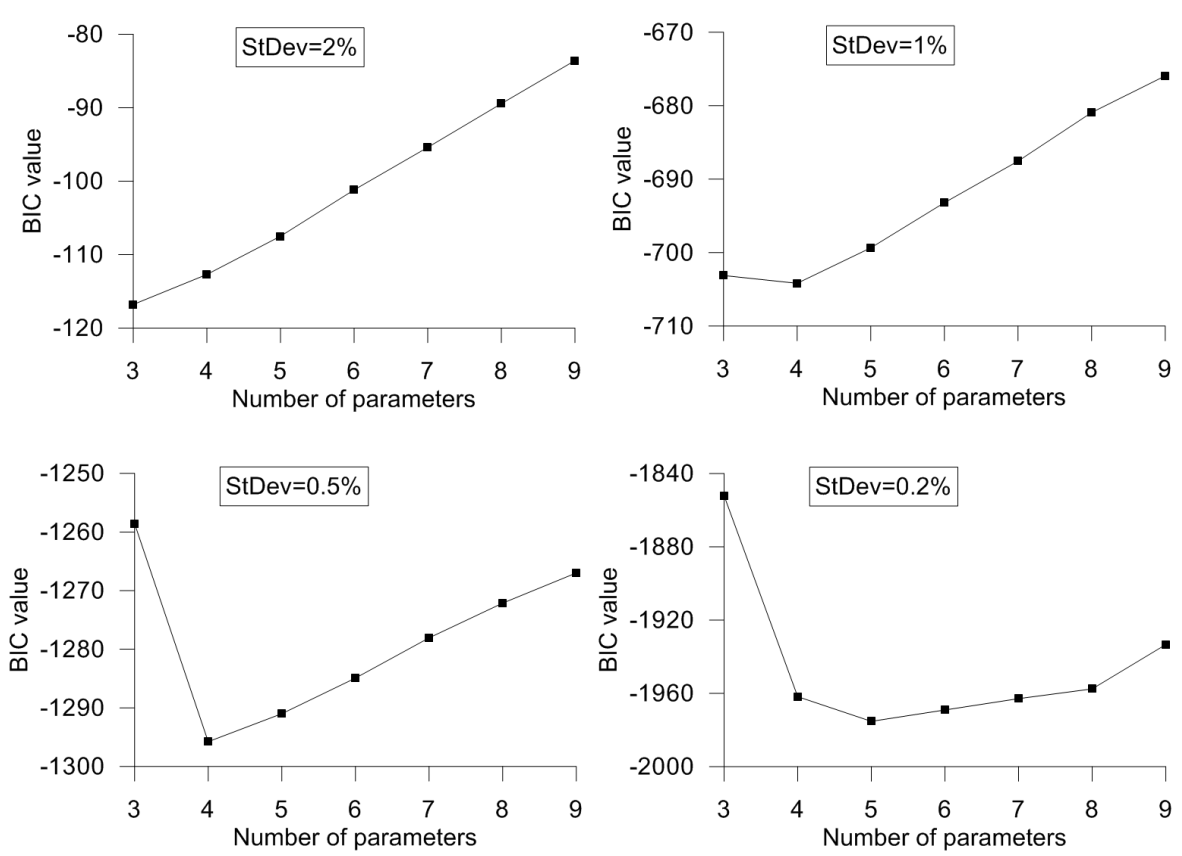

Fig. 3. BIC values for different levels of random noise for 3-layers model.

The next step was an analysis of obtained BIC values for each dataset to simulate decisions that should be made for real data without knowledge of the exact number of parameters.

For the 3-layered model (Fig. 3), analysis was conducted for 4 levels of random noise added. Noise with the standard deviation of $2 \%$ make model composed of 3 parameters optimal (all 3 layers isotropic and without gradient). If the noise drops to $1 \%$ or $0.5 \%$ velocity gradient in the middle layer should be considered as a forth parameter. Model including elliptical anisotropy coefficient is justified only if the noise has standard deviation equal $0.2 \%$ or less.

Analysis for the 5-layered model (Fig. 4) has shown that for the noise level of $0.2 \%$ or more, the additional parameters of forth layer are not justified and the model composed of 5 velocities is sufficient. Random noise not exceeding $0.1 \%$ gives basis to consider model with elliptical anisotropy and gradient in forth layer. 

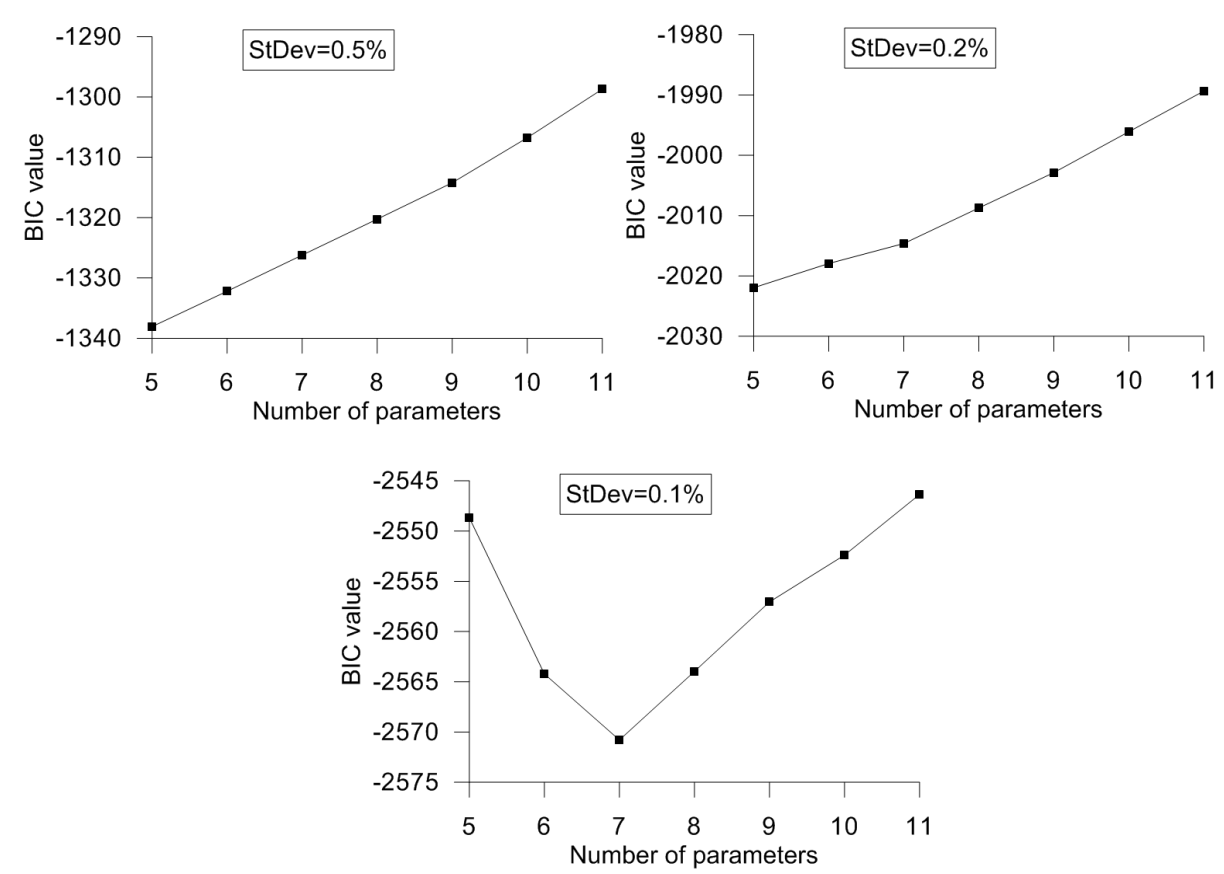

Fig. 4. BIC values for different levels of random noise for 5-layers model.

\section{Conclusions}

The proposed method for the inversion of the velocity model with assumption of elliptical anisotropy and vertical gradient allows one to obtain model with given number of velocity parameters. Additional parameters can improve similarity of modelled and measured traveltimes but with the low-quality data they may not carry useful information and make model too complex. The BIC analysis can help to choose optimal model parameterization for the specific dataset.

The analysis carried out on the synthetic data has shown that the method of velocity model optimization gives results consistent with the expected model. Knowing the exact traveltimes and location of sources and receivers (the scheme of vertical seismic profiling) one can use multiple sources inversion without problems with the solution stability.

Basing on the BIC analysis we can formulate two general conclusions. Firstly, the lower quality of data, the less parameters are adequate to describe the velocity model. With decreasing level of noise added to synthetic dataset minimum of BIC value is achieved by model with greater number of parameters (Figs. 3 and 4). Secondly, the greater total number of parameters in genuine model (i.e. number of layers), the lower level of noise allowing one to introduce additional parameter like gradient or anisotropy. The reason is that more parameters can compensate lack of this value and reduce the target function value.

This research was supported by AGH University of Science and Technology, Faculty of Geology, Geophysics and Environmental Protection as a part of the project No.15.11.140.194

\section{References}

1. M. A. Slawinski, C. J. Wheaton, M. Powojowski, Geophysics 69, 373-377 (2004) DOI: $10.1190 / 1.1707055$ 
2. P. F. Daley, F.Hron, Geophysics 44, 27-38 (1979) DOI: 10.1190/1.1440920

3. L. Thomsen, Geophysics 51, 1954-1966 (1986) DOI: 10.1190/1.1442051

4. R. Brown, M. Lamoureux, M. Slawinski, R. Slawinski,. Direct traveltime inversion of VSP data for elliptical anisotropy in layered media. CREWES Research Report (2000)

5. Y. Rogister, M. Slawinski, Geophysics 70, 37-41 (2005) DOI: 10.1190/1.2049347

6. J. A. Nelder, R. Mead, Comput. J. 7, 308-313 (1965) DOI: 10.1093/comjnl/7.4.308

7. J. H. Mathews, K. D. Fink, Numerical Methods Using Matlab (Prentice Hall, New Jersey, 2004)

8. R. Kass, A. Raftery, J. Am. Stat. Assoc. 90, 773-395 (1995) DOI: 10.1080/01621459.1995.10476572

9. M. B. Priestley, Spectral Analysis and Time Series (Academic Press. New York, 1981)

10. T. Danek, M. A. Slawinski, Geophysics 77, 239-243 (2012) DOI: 10.1190/geo20120051.1 